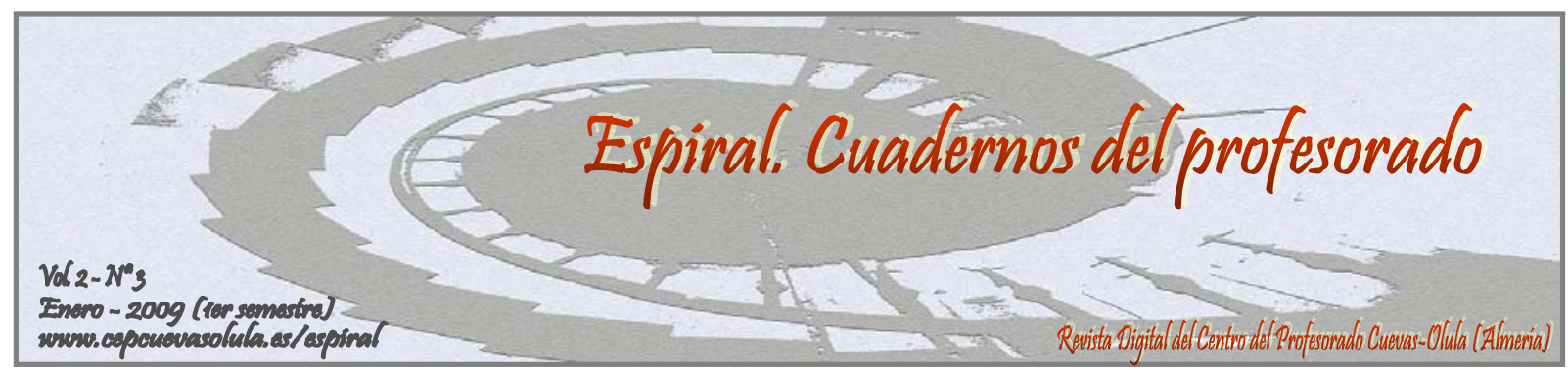

\title{
LAS TECNOLOGÍAS DE LA INFORMACIÓN Y LA COMUNICACIÓN (TIC) Y LAS COMPETENCIAS BÁSICAS EN EDUCACIÓN
}

\author{
INFORMATION AND COMMUNICATION TECHNOLOGY (ICT) AND BASIC \\ COMPETENCIES EDUCATION
}

\section{Francisco José Martínez López}

\section{Profesor de Tecnología. I.E.S. Ricardo Ortega, Fuente Álamo, Región de Murcia (España)}

Martínez López, F. J. (2009). Las tecnologías de la información y la comunicación (TIC) y las competencias básicas en educación [en línea]. Espiral. Cuadernos del Profesorado, 2(3), 15-26. Disponible en: http://www.cepcuevasolula.es/espiral.

Enviar correspondencia a: fjmartinezlopez@gmail.com

RESUMEN: Estamos ante una nueva sociedad, la Sociedad de la Información, fruto del impulso de las nuevas tecnologías dominantes, las Tecnologías de la Información y la Comunicación (TIC). El sistema educativo tiene que dar respuesta a las nuevas demandas de la población para satisfacer sus necesidades. Un análisis cronológico nos acerca a la necesidad de definir unas competencias básicas que debe poseer todo ciudadano. Diferentes puntos de vista e intereses se pondrán en juego. El papel de las TIC será crucial en el logro de la competencia digital, dentro de las competencias básicas que debe poseer todo ciudadano, para facilitar el que pueda ejercer sus derechos y deberes con plenitud, acordes con la sociedad cambiante a la que han de ir constantemente adaptándose.

Palabras clave: tecnología de la información y la comunicación, competencias básicas, sociedad de la información, sociedad digital, educación.

ABSTRACT: We face a new society, the Information Society, the result of the momentum of new technologies, Information and Communication Technologies (ICT). The education system must respond to new demands of the population to meet their needs. An analysis about the time we need to identify basic skills that every citizen should have. Different views and interests will be at stake. ICT will be crucial in achieving competitive digital, within the basic competencies that every citizen should have, to facilitate that it can exercise their rights and duties fully in line with the changing society, to which must be constantly adapted.

Key words: Information and Communication Technologies, basic competencies, information society, digital society, education. 


\section{1.- INTRODUCCIÓN.}

El final del siglo XX, y los inicios del presente, nos están adentrando vertiginosamente en una verdadera revolución tecnológica, que con el paso de los años y la perspectiva que nos ofrece, nos hará reconocerlo como un auténtico acontecimiento histórico: la sociedad de la información, al menos tan importante como lo fue la revolución industrial de finales del siglo XVIII, que daría paso a la sociedad industrial. Se trata de una verdadera revolución, que como señala Gimeno Perelló (2005):

“...una característica de esta revolución es su capacidad de penetración en todos los dominios de la actividad humana, penetración que incluso orienta el proceso de cualquier actividad, induciendo nuevos comportamientos, nuevos conocimientos, costumbres $y$ hábitos nuevos, nuevos valores o nuevos productos" (p. 215).

Nos hallamos ante una sociedad de la información emergente, impulsada por un desarrollo vertiginoso de las Nuevas Tecnologías de la Información y la Comunicación (TIC), fruto de los grandes desarrollos y avances científicos y tecnológicos que en un marco universal y globalizador integran y afectan a todas las facetas de nuestra vida. Ello conlleva una modificación de nuestros hábitos, de la actividad laboral, social y cultural, pero también la educativa, por supuesto. Estamos pues, trastocando las formas de enseñar y de aprender, los medios empleados y la forma de utilizarlos y entenderlos.

Pero, ¿qué entendemos por sociedad de la información? Si las nuevas tecnologías de la información y la comunicación calan en todas las facetas de nuestra vida, también en las educativas, cabría preguntarnos ¿cómo inciden en la educación? En definitiva, son bastantes las cuestiones que se nos presentan al cuestionarnos si realmente tenemos el camino claramente identificado y definido ante los nuevos retos que se nos presentan con las TIC, o simplemente habremos de ir labrándolo, de manera constante y progresiva, sin perder la perspectiva de unos objetivos que, obedeciendo a nuestros intereses educativos hagan de horizonte.

\section{2.- LA SOCIEDAD DE LA INFORMACIÓN.}

Puede ser bastante complicado dar respuesta a lo que se entiende por sociedad de la información. De hecho, no podemos hacerlo de manera unívoca, especialmente cuando confluyen y se superponen elementos de muy diversa índole. Sin embargo, sí podríamos intentar una aproximación analizando el término sociedad, el cual nos lleva a un colectivo de personas, pueblos o naciones, que se agrupan para cumplir con una finalidad, a través de la mutua cooperación.

Haciendo una revisión histórica de la evolución de nuestra sociedad (figura 1), como considera Abad Amorós (2005), podríamos identificar inicialmente a la sociedad moderna que a finales del siglo XVIII y siglo XIX, tras el inicio de la revolución industrial deja paso a la sociedad industrial, en la que todos sus miembros tienen acceso, tanto a los bienes que ellos mismos producen como a los producidos por terceros, para abrir el camino a la sociedad postindustrial, tras la segunda guerra mundial, en la que sus componentes tienen acceso a los servicios prestados por otros y que facilitará el camino al modelo de sociedad de la información, basado en la producción de servicios, frente a la producción de productos (Bell, 1991).

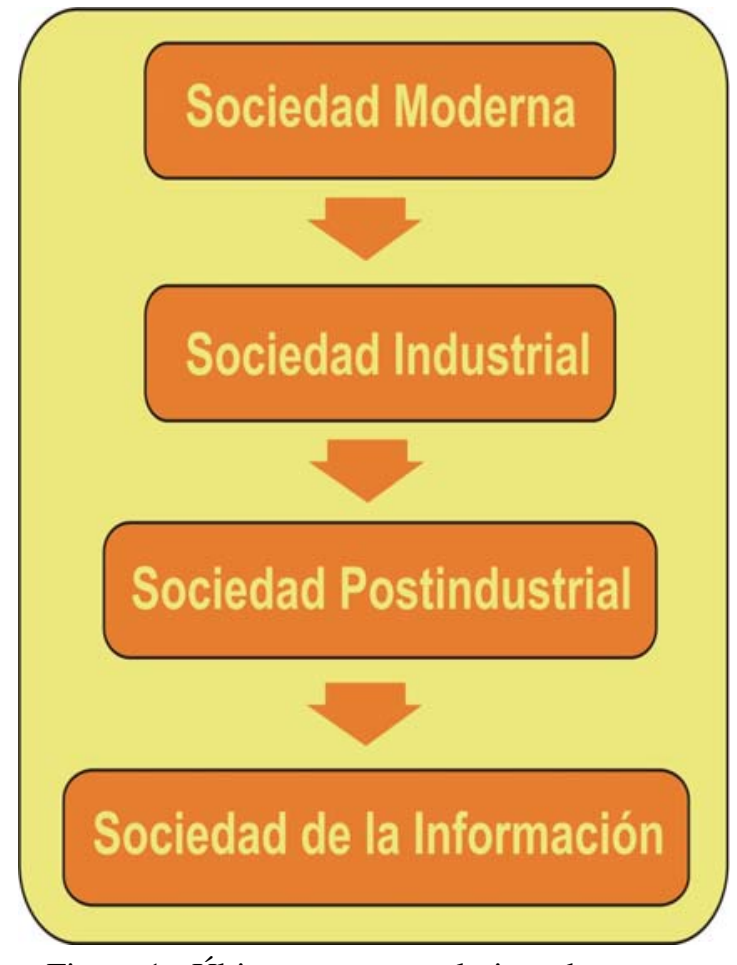

Figura 1.- Últimas etapas evolutivas de nuestra sociedad. Fuente: elaboración propia.

Aproximándonos a este modelo señalado por Bell, conviene recordar la definición que ofrece Castells (1998), mencionado por Abad Amorós (2005), que entiende a la sociedad de la información como: 
"Nuevo sistema tecnológico, económico y social. Una economía en la que el incremento de productividad no depende del incremento cuantitativo de los factores de producción (capital, trabajo, recursos naturales), sino de la aplicación de conocimientos e información a la gestión, producción y distribución, tanto en los procesos como en los productos" (p. 251-252).

Alejándonos del matiz económico, que como sistema se le da a la sociedad de la información, se pronuncian otros autores como Linares (1995) cuando señala que se basa en el conocimiento y en los esfuerzos en convertir la información en conocimiento, siendo la necesidad de estos esfuerzos mayores cuando mayor es la información generada, estableciendo además otra dimensión, la de la velocidad con la que la información se genera, transmite y procesa.

Nosotros podemos definir a la sociedad de la información como el entorno que, resultante del eficaz y optimizado desarrollo de las nuevas tecnologías de la información y la comunicación, busca la satisfacción de las necesidades humanas de sus miembros.

Esta sociedad de la información no debería ser confundida por la sociedad del conocimiento, más específica y restrictiva, al considerar que tener información no equivale necesariamente tener conocimiento. Ya que conocer y pensar no es simplemente almacenar, tratar y comunicar datos, sino que se trata del resultado de un proceso en el que se selecciona la información y analiza desde una postura reflexiva que busca la profundización en la creación del mensaje.

Pero, ¿qué es lo que realmente se espera de la sociedad de la información? En este sentido podría contribuir el artículo 1 de la Declaración de Principios emanados de la Cumbre Mundial de la Sociedad de la Información celebrada por la ONU en 2003:

“...declaramos nuestro deseo y compromiso comunes de construir una sociedad de la información centrada en la persona, incluyente y orientada al desarrollo, en la que todos puedan crear, consultar, utilizar y compartir la información y el conocimiento, para que las personas, las comunidades y los pueblos puedan desarrollar su pleno potencial en la promoción de su desarrollo sostenible y mejorar su calidad de vida..."

Por tanto, nos enfrentamos ante la necesidad de una revisión profunda de la razón de ser de las instituciones educativas, las formas de enseñar y aprender, la forma de organizarse ante los nuevos y apasionantes cambios que de manera un tanto acelerada e incesante se van produciendo. No sirve detenerse o relajarse, si no es para reflexionar sobre la forma de abordar el reto que nos supone la incursión de las Nuevas Tecnologías (NNTT) en el sistema educativo.

\section{3.- LA SOCIEDAD DIGITAL $Y$ SU INCIDENCIA.}

Considerando la sociedad de la información y desde el punto de vista educativo, se nos presenta un nuevo entorno, llamémosle digital, que nos incorpora velozmente al uso indiscriminado y potencialmente masivo de las NNTT. Podríamos pensar incluso que estamos ante un proceso de innovación educativa acelerada. Sin embargo, no se trata de su uso, un tanto obligado para sobrevivir en el nuevo entorno social y globalizador, en el que nos vamos sumergiendo a pasos agigantados, como de la utilización bajo unos parámetros óptimos de reflexión sobre ellas. Innovar, desde el punto de vista educativo, requerirá bastante al docente, especialmente formación, rigor y trabajo colaborativo. Se ha de generar el entorno óptimo para enseñar a los alumnos y alumnas a hacer, en lugar de a responder preguntas sobre cómo hacer (Díaz del Valle y Sánchez Baena, 2004). En definitiva, debe de producirse un dinamismo que pretenda alcanzar a la sociedad del conocimiento desde la propia sociedad de la información.

¿Cómo se nos manifiesta la sociedad digital? Cebrián (1999), citado por Benito Morales (2000), resume en seis puntos algunas evidencias sobre la sociedad digital, señalando que es global, entendida como que no conoce fronteras geográficas ni temporales, a pesar de que aún existan bastantes reticencias en este sentido, es convergente, confluyendo en ella multitud de disciplinas que tradicionalmente se han desarrollado por separado, es interactiva, basada en la cooperación y el diálogo, es caótica, no admitiendo jerarquías ni encorsetamientos que limiten su movilidad, es la cuna de una nueva realidad virtual y finalmente es rauda, desarrollándose de forma casi autónoma a gran velocidad, desbordando todas las previsiones sobre sus previsiones de crecimiento. 
En este marco y ante las reacciones posibles por parte de los centros docentes para adaptarse a las TIC y al nuevo contexto cultural, Aviram (2002) identifica tres posibles escenarios:

$1^{\circ}$. Un escenario tecnócrata, identificado simplemente por el hecho de que las escuelas se adaptan realizando pequeños ajustes: en primer lugar la introducción de la "alfabetización digital" de los estudiantes en el curriculum para que utilicen las TIC como instrumento para mejorar la productividad en el proceso de la información "aprender sobre las TIC" y luego progresivamente el empleo de las TIC como fuente de información y proveedor de materiales didácticos, lo que podríamos denominar como “aprender de las TIC”. En definitiva, que las escuelas sobrevivirán a la revolución de las TIC, simplemente con unos cambios mínimos en los curricula.

$2^{\circ}$. Otro escenario reformista, que englobaría además de los dos niveles de integración de las TIC, ya mencionados anteriormente, un tercero "aprender con las TIC”, es decir, la introducción en las prácticas docentes de nuevos métodos de enseñanza/aprendizaje constructivistas que contemplen el uso de las TIC como instrumento cognitivo.

$3^{\circ}$. Y finalmente un escenario holístico: donde los centros hacen una apuesta a fondo, llevando a cabo una profunda reestructuración de todos sus elementos.

Estos tres posibles escenarios representan también tres formas diferentes de enfocar la incidencia del entorno digital ofrecido por las TIC en la educación. Son tres formas distintas de actuar, conducentes por otra parte a políticas totalmente diferentes.

En la línea del escenario holístico se pronuncian algunos autores como Majó (2003):

"la escuela y el sistema educativo no solamente tienen que enseñar las nuevas tecnologías, no sólo tienen que seguir enseñando materias a través de las nuevas tecnologías, sino que estas nuevas tecnologías aparte de producir unos cambios en la escuela producen un cambio en el entorno y, como la escuela lo que pretende es preparar a la gente para este entorno, si éste cambia, la actividad de la escuela tiene que cambiar. Por lo tanto, debe irse más allá de la enseñanza de las nuevas tecnologías y de la enseñanza a través de ella".

En definitiva, sea cual sea la respuesta del sistema educativo, se hace necesaria, aún cuando ésta pueda oscilar en cuanto a su línea adoptada. El ritmo imparable de los avances tecnológicos en las nuevas formas de comunicar e informar representa un impulso constante y provocador ante el que irremediablemente sucumbimos.

Es por ello por lo que se necesitan nuevos conocimientos y competencias como respuesta a los profundos cambios socio-económicos que originan los nuevos instrumentos tecnológicos y la globalización económica y cultural. Se trata de conseguir una formación continua a lo largo de la vida para todos los ciudadanos, para lo que se precisará de la incorporación de una alfabetización digital básica (cada vez más necesaria, a la vez que imprescindible para todo ciudadano) y diversos contenidos relacionados con el uso específico de las TIC en diversos ámbitos, presentando éstos en los centros educativos funcionalidades tales como: gestión y administración del centro (secretaría, gestión de la tutoría de alumnos y alumnas, biblioteca...), alfabetización digital de los estudiantes, además de los/as profesores/as y familias, uso didáctico para facilitar los procesos de enseñanza y aprendizaje, comunicación con las familias (a través de programas de gestión de faltas de asistencia, de la Web del centro...), comunicación con el entorno, relación entre profesores de diversos centros compartiendo recursos o formando comunidades virtuales (Marquès Graells, 2000a),...

Pero, nos deberíamos preguntar cómo enfrentarnos al reto continuo al que nos impulsan las NNTT, ¿qué competencias hemos de adquirir?, ¿cómo podemos definir las competencias en TIC?, ¿cuáles son?

\section{4.- LAS COMPETENCIAS. ANTECE- DENTES.}

Existen una amplia variedad de términos que aunque con diferentes denominaciones (aprendizajes básicos, necesidades básicas de aprendizaje, competencias básicas,...) son bastante coincidentes en su significación, haciendo referencia a conocimientos, habilidades y valores relacionados con la autonomía en la vida para ejercer con eficacia los derechos y deberes ciudadanos. 
Algunos autores como Chomsky (1965) ya se anticipan al término competencia, introduciendo el concepto de competencia lingüística al referirse a la gramática generativa. Distingue entre los conceptos de "competence" o capacidad general aplicable en ocasiones múltiples, y la "performance", entendida como habilidad que se requiere para resolver una situación puntual. También se hace referencia a la competencia cognitiva al hablar de las teorías cognitivas del aprendizaje dentro del contexto de las inteligencias múltiples.

La Ley Orgánica 5/2002 de 19 de junio, de las Cualificaciones y de la Formación Profesional define competencia profesional como "el conjunto de conocimientos y capacidades que permiten el ejercicio de la actividad profesional conforme a las exigencias de la producción y el empleo".

La competencia es sin duda un concepto complejo, pero que asociado al mundo profesional y desde un punto de vista, un tanto reduccionista, es sinónimo de capacidad, idoneidad, habilidad o maestría, sin embargo debe conllevar algo más, debe corresponderse con un saber actuar para articular, componer, dosificar e integrar los recursos adecuados con la finalidad de obtener un resultado satisfactorio e idóneo ante situaciones diversas.

Laisner (2000) define las competencias como un saber hacer complejo, resultado de la integración, movilización y adecuación de capacidades, conocimientos, actitudes y habilidades, utilizados eficazmente en situaciones que tengan un carácter común. En esta definición Sarramona (2008) eliminaría la adecuación de capacidades ajustándola aún más.

Por otra parte, también se definen otros términos con cierta similitud, como en la Declaración Mundial sobre "Satisfacción de las necesidades básicas de aprendizaje" de la Conferencia Mundial sobre Educación para todos de Jomtien (1990), patrocinada por el Banco Mundial, el PNUD, la UNESCO, la UNICEF y el FNUAP, presenta un consenso sobre la educación básica ampliada y establece como objetivo la satisfacción de las necesidades básicas de aprendizaje, para lo que cada persona -niño, joven o adulto- deberá estar en condiciones de aprovechar las oportunidades educativas ofrecidas para satisfacer dichas necesidades, abarcando tanto las herramientas esenciales para el aprendizaje como los contenidos básicos del mismo. Además considera a la educación básica como la base para un aprendizaje y un desarrollo humano permanentes sobre el cual se puedan ir asentando nuevos niveles y tipos de educación y capacitación, en definitiva más que un fin en sí misma, precisando para ello una visión ampliada que vaya más allá de los planes de estudios del momento y de los sistemas tradicionales de instrucción.

En el Foro Consultivo Internacional sobre Educación para Todos de Amman (1996) se realiza un examen sobre los progresos alcanzados en la puesta en práctica de los objetivos de la educación para todos de Jomtien. Se habla de algunos avances pero también se sugiere premura en algunas acciones ya que el progreso en las mismas había sido mucho más lento de lo esperado.

El Foro Mundial sobre la Educación de Dakar (2000) reafirma que toda persona tiene derecho a beneficiarse de una educación concebida para dar respuesta a sus necesidades educativas fundamentales, que le permita aprovechar a cada persona su potencial, con el fin de permitir una vida mejor y transformar la sociedad en la cual viven.

Con anterioridad, la Organización para la Cooperación y el Desarrollo Económico (OCDE) crea a finales de 1997 un proyecto denominado DeSeCo (Definition and Selection of Competencies) para servir como fuente de información para identificar las competencias clave y fortalecer las encuestas internacionales que midan el nivel de competencia de jóvenes y adultos. Define a las competencias como:

"la capacidad para responder a las demandas complejas y llevar a cabo tareas de forma adecuada. Cada competencia se construye a través de la combinación de habilidades cognitivas y prácticas, conocimiento (incluyendo el conocimiento tácito), motivación, valores, actitudes, emociones y otros componentes sociales y conductuales".

El Proyecto PISA (Program for International Student Assesment) de la OCDE realizado por encargo de los gobiernos e instituciones educativas y centrado en estudiantes de una determinada edad y no de un nivel escolar específico, plantea la necesidad de contar con datos regulares y comparables, que permitan derivar un conjunto de indicadores básicos que proporcionarán a los políticos y administradores 
de la educación un perfil de los conocimientos, destrezas y competencias de los estudiantes de cada país, así como un conjunto de indicadores contextuales que se espera clarifiquen las relaciones entre dichas destrezas y las variables demográficas, sociales, económicas y educativas más importantes. Prestando especial interés en la lectura, matemáticas y ciencias, e incluyendo por lo menos una competencia intracurricular en cada evaluación, siendo las seleccionadas para las dos primeras evaluaciones el aprendizaje y la solución de problemas autorregulados. El objetivo es evaluar hasta qué punto los jóvenes han adquirido los conocimientos que les servirán en su vida adulta.

La Red Europea de Información en Educación (EURYDICE) (2002), considera la necesidad de llevar a cabo un enfoque europeo común para identificar las competencias que todos los ciudadanos deben conseguir, llevando a cabo un estudio sobre las competencias clave, para establecer como se hallan desarrolladas, cómo se evalúan, y mostrar la amplia gama de características y de terminología con que se asocian. Se considera de una gran preocupación el impacto que una adquisición desequilibrada de las mismas pueda estar ocasionando sobre la justicia social y económica, pudiendo conducir a la marginalización $\mathrm{y}$, en último término, a la exclusión social. A ello se une una cierta idea de que se está centrando demasiado a las competencias clave en el trabajo y no tanto en su importancia para lograr una vida personal satisfactoria.

Pero ¿qué entendemos por las competencias clave? No existe una definición única, sino que tenemos un amplio abanico de posibilidades en cuanto a su definición, donde cualquiera de ellas vendrá impregnada por la formación científica y el papel que juegue en la sociedad su autor/a. Por lo que podríamos generalizar señalando que no existe una acepción universal del concepto de competencia clave, aunque sí podríamos ajustarnos al atributo de clave, fundamental o básico, como que debe ser necesaria y beneficiosa para cualquier individuo y para la sociedad en su conjunto, mostrándose eficaz para el individuo, tanto en situaciones que le sean conocidas como en otras que puedan serle imprevisibles, permitiendo además la actualización continua de sus conocimientos y destrezas.

La Cumbre Extraordinaria del Consejo de Europa de Lisboa (2000), reconociendo que la adaptación a la globalización, y al desplazamiento hacia las economías basadas en el conocimiento, es un reto europeo, propone la identificación de las competencias básicas para garantizar el acceso al aprendizaje en la sociedad de la innovación y el conocimiento, haciendo hincapié en que todo ciudadano deberá poseer la formación necesaria para vivir y trabajar en la sociedad de la información y en que desde un marco europeo se deberían definir unas nuevas cualificaciones básicas que fuesen proporcionadas a través de la formación continua.

En la Cumbre del Consejo Europeo de Barcelona (2002) se desarrolla el programa de trabajo “Educación y formación 2010” (ET2010) apostando por una economía competitiva basada en el conocimiento, insistiendo en la mejora del dominio de las competencias básicas, intensificando la enseñanza de las lenguas extranjeras desde una edad muy temprana y el desarrollo de la cultura digital, entre otros, para promover una dimensión europea en la enseñanza.

Con un mayor grado de concreción, al ubicar el momento de la adquisición, Castellano González (2006) concibe las competencias básicas como "el conjunto de habilidades cognitivas, procedimentales y actitudinales que pueden y deben ser alcanzadas a lo largo de la enseñanza obligatoria por todo el alumnado, respetando las características individuales", señalando además que resultan imprescindibles para asegurar su desenvolvimiento personal y social, al igual que para una ejercitación efectiva de sus derechos y deberes ciudadanos.

Podríamos concluir que:

a) Representan una capacidad de respuesta satisfactoria e idónea a situaciones reales diversas.

b) No son, en sí mismas, conocimientos, habilidades/destrezas o actitudes que puedan desarrollarse por separado, sino que se trata de una combinación de todas ellas.

c) Pueden y deben ser adquiridas durante la enseñanza obligatoria.

d) La aplicación de las competencias básicas se manifiesta mediante el desarrollo de procesos mentales complejos que permiten la adopción de actuaciones adaptadas a situaciones concretas $\mathrm{y}$ reales. 
e) Deben ligarse, entre otros, al desarrollo de una cultura digital.

\section{5.- LAS TIC Y LAS COMPETENCIAS BÁSICAS.}

Analizada la trayectoria que ha ido teniendo durante estos últimos años la necesidad del establecimiento de un nuevo diseño curricular que contemple unas competencias básicas, como elemento imprescindible para permitir la formación de unos/as ciudadanos/as, que puedan ejercer con plenitud sus deberes y derechos, como integrantes de la nueva sociedad en la que se hallan inmersos, se hace, por tanto, precisa la necesidad de replantearse los curricula como elementos esenciales que intervengan activamente en la formación de los ciudadanos de un país, extrayendo su capacidad crítica para ver el mundo, transformar y adaptarse a los cambios de la sociedad. Se trata de establecer un enfoque más globalizador e integrador en la sociedad de manera que reaccione satisfactoriamente a las necesidades cambiantes de su realidad.

Señala López (2006) la importancia de insistir en la educación obligatoria, tanto en las herramientas esenciales del aprendizaje con la expresión oral y escrita, comprensión lectora y la resolución de problemas, como en los contenidos fundamentales para la adquisición de capacidades, actitudes y valores.

En esta línea la Comisión Europea de Educación establece unas competencias clave o destrezas básicas necesarias para el aprendizaje del individuo a lo largo de su vida, considerando que en ellas se sustenta el desarrollo personal, la inclusión social y la ciudadanía activa y el empleo, animando además a los estados miembros a que dirijan sus políticas en esta dirección.

Las competencias clave a las que hace referencia el documento sobre "Competencias clave para el aprendizaje permanente, un marco de referencia europeo (2005)", son las siguientes:

1. Comunicación en lengua materna.

2. Comunicación en lenguas extranjeras.

3. Competencia matemática y competencias básicas en ciencia y tecnología.

4. Competencia digital.

5. Aprender a aprender.

6. Competencias interpersonales, interculturales y sociales, y competencia cívica

7. Espíritu de empresa.

\section{Expresión cultural.}

Donde las competencias se definen como una combinación de conocimientos, capacidades y actitudes adecuadas al contexto, siendo consideradas clave porque todo ciudadano las precisa para su realización y desarrollo personal, así como para el desarrollo de la ciudadanía activa, la inclusión social y el empleo.

Dentro de las competencias clave propuestas por la Comisión del Parlamento Europeo figura la competencia digital que define como:

"La competencia digital entraña el uso seguro y crítico de las Tecnologías de la Sociedad de la Información (TSI, en adelante) para el trabajo, el ocio y la comunicación. Se sustenta en las competencias básicas en materia de TSI: el uso de ordenadores para obtener, evaluar, almacenar, producir, presentar e intercambiar información y comunicarse y participar en redes de colaboración a través de Internet".

Esta competencia exige por tanto unos importantes conocimientos sobre las TSI en situaciones cualesquiera de la vida (profesional, privada...) como el manejo de hojas de cálculo, bases de datos, almacenamiento y gestión de la información, tratamiento de textos, además de aprovechar las oportunidades que ofrece internet y las comunicaciones por medios electrónicos.

Además el individuo necesitará estar capacitado para buscar, obtener y tratar información, utilizarla de forma crítica, sistematizada y pertinente.

En este sentido la L.O.E. (2006), precedida por la L.O.C.E. (2003) ajena a estas nuevas líneas de afrontar la educación, recoge estas nuevas demandas y establece una modificación de la definición de curriculum enriqueciéndolo con un nuevo elemento, quedando en su art. 6. como " $\mathrm{el}$ conjunto de objetivos, competencias básicas, contenidos, métodos pedagógicos y criterios de evaluación de cada una de las enseñanzas reguladas en la presente ley", también en su art. 20.2 (capítulo II: Educación Primaria) establece que "el alumnado accederá al ciclo educativo o etapa siguiente siempre que se considere que ha alcanzado las competencias básicas correspondientes y el adecuado grado de madurez", en el art. 26.2 (capítulo III: educación secundaria obligatoria) se señala que "se prestará una atención especial a la adquisición y 
el desarrollo de las competencias básicas y se fomentará la correcta expresión oral y escrita y el uso de las matemáticas”, además determina en el art. 31 que "los alumnos que al terminar la Educación Secundaria Obligatoria hayan alcanzado las competencias básicas y los objetivos de la etapa obtendrán el título de Graduado en Educación Secundaria Obligatoria”.

Las 8 competencias básicas establecidas en la L.O.E. son las siguientes:

1. Competencia en comunicación lingüística.

2. Competencia matemática.

3. Competencia en el conocimiento y la interacción con el mundo natural.

4. Tratamiento de la información y competencia digital.

5. Competencia social y ciudadana.

6. Competencia cultural y artística.

7. Competencia para aprender a aprender.

8. Autonomía e iniciativa personal.

Estas se hallan entrelazadas de forma que se solapan abordando enfoques que se complementan entre sí urdiendo en su conjunto una combinación de conocimientos, capacidades y actitudes, que todas las personas precisan para su realización y desarrollo personal en la sociedad actual. La adquisición de las competencias básicas no es exclusiva de un área de conocimiento determinada, por lo que pueden trabajarse en todas las áreas.

Concretando en el tratamiento de la información y competencia digital, esta competencia se agruparía en el ámbito de la comunicación y la expresión, conjuntamente con la competencia lingüística, la matemática y la cultural y artística, y precisaría de unos conocimientos, habilidades y usos racionales para el empleo pertinente de los códigos de los diferentes sistemas. Así pues, las TIC se presentan como un amplio y poderoso instrumento de aprendizaje, capacitando al alumnado para la toma de decisiones, la resolución de problemas reales, la utilización de la comunicación en entornos colaborativos y para la participación en comunidades de aprendizaje, formales e informales. Para ello debe adquirir unos conocimientos que estarán vinculados a los modos de operar de los sistemas tecnológicos, el manejo básico de las redes en general e Internet, los servicios que éste permite aprovechar y los riesgos y protecciones para garantizar la seguridad en su uso.

Marqués Graells (2000b, p. 4) señala como competencias básicas en TIC que deben configurar la denominada alfabetización digital (computer literacy) para todos los ciudadanos, las siguientes:

$\checkmark$ Conocimiento básico del sistema informático: elementos del hardware, tipos de software, redes...

$\checkmark$ Gestión básica del equipo: administración de archivos y carpetas, antivirus...

$\checkmark$ Uso del procesador de textos: correctores...

$\checkmark$ Navegación en Internet: búsqueda y selección de información, telegestiones...

$\checkmark$ Uso del correo electrónico.

$\checkmark$ Creación, captura y tratamiento de imagen digital.

$\checkmark$ Elaboración de documentos multimedia: presentaciones, páginas Web.

$\checkmark$ Conocimiento básico de la hoja de cálculo y las bases de datos.

En cualquier caso, hemos de tener en cuenta el hecho de hallarnos en una sociedad de la información en continua evolución en donde irán apareciendo nuevos recursos TIC que influirán significativamente en ella y por ende en el mundo educativo y ante este panorama las competencias digitales habrán de ir sufriendo una evolución constante que requerirá de una actualización continua debiendo verse reflejada en las intervenciones educativas sucesivas.

En este sentido, Marqués Graells (2008) apunta seis claves para el éxito de una buena integración de las TIC:

1) Infraestructuras TIC en las aulas, en el centro, en la casa... Si no se dispone de unas TIC adecuadas se estará dificultando la integración de las mismas en los procesos de e-a, y por tanto el que el/la alumno/a adquiera la competencia digital deseada.

2) Recursos digitales de apoyo. Tanto el hardware, como el software, han de estar en consonancia para un desarrollo adecuado de las actividades, de forma que se posibiliten los aprendizajes deseados. Habrá que prever procesos de búsqueda, análisis e integración de los recursos digitales en las actividades de aprendizaje. 
3) Coordinación TIC. Infraestructuras TIC, pero también mantenimiento y coordinación se hacen necesarios para facilitar la integración satisfactoria de las TIC.

4) Apoyo del equipo directivo y compromiso de la comunidad educativa. La actuación firme y decidida desde los equipos de dirección con el compromiso de la comunidad educativa del centro reflejada en el PEC contribuirá sensiblemente en la evolución en cuanto al desarrollo de las TIC

5) Formación del Profesorado. En este sentido Noriega Álvarez y Muñoz González (1996) señalan lo que se denominaría ligaduras refiriéndose a aquellas circunstancias que limitan la iniciativa y la creatividad y que ponen límites al desarrollo de un proyecto, mencionándose entre ellas las ligaduras tecnológicas, puesto que limitan la realización de proyectos que supongan el uso de medios o recursos tecnológicos de los que se carece o se desconoce. Si a ello sumamos lo que para nuestra sociedad supone los vertiginosos avances en el terreno de las TIC y la necesidad de que nuestros/as alumnos/as inmersos en un contexto socio-económico y tecnológico en continuo y acelerado cambio, hemos de ser conscientes que infraestructuras y recursos son una condición necesaria, pero no suficiente para lograr la integración de las TIC. Debe existir una formación que ha de estar convenientemente graduada.

6) La actitud del profesorado ante el uso didáctico de las TIC. En la situación actual, en la que solamente utilizan las TIC con cierta frecuencia en las aulas un $20 \%$ de los docentes, según el Foro TICEMUR 2008, hemos de tener en cuenta la predisposición del docente como un parámetro primordial e influyente, y que lo será tanto mayor cuanto más amplia sea su formación instrumental-didáctica, repercutiendo en una actitud positiva hacia la innovación con las TIC de manera que pueda facilitar su aplicación en su práctica docente.

\section{6.- DISCUSIÓN.}

Hemos podido analizar la inexistencia de unanimidad a la hora de mostrar el concepto sobre lo que entendemos por sociedad de la información, lo que lejos de empobrecerlo lo enriquece ofreciéndole una mayor versatilidad, al mostrarnos una generosidad que se distancia de una visión exclusivista. Numerosos intereses de carácter colectivo (gubernativo, social...) e incluso personales nos llevarán a derroteros, aparentemente desligados, pudiendo primar el carácter económico, entre otros, que pone su enfoque en la productividad como elemento notorio y distintivo, mientras que otros autores optan por una línea dirigida al conocimiento, a los esfuerzos en convertir la información en conocimiento, siendo la necesidad de estos esfuerzos mayores cuando mayor es la información. Aunque sin llegar a entender la sociedad de la información como la sociedad del conocimiento.

Por otro lado, se detecta una preocupación internacional por asentar las bases para una educación que, puesta al día, permita satisfacer las necesidades básicas de aprendizaje. Se evidencia un gran interés en definir unas líneas que, involucrando un amplio reconocimiento, sirvan de hilo conductor, permitiendo el desarrollo de actuaciones enfocadas a dar respuestas a las necesidades educativas actuales. Cada persona debe saber aprovechar su potencial, ante la nueva realidad de la sociedad de la información con el fin de alcanzar una vida mejor y transformar la sociedad en la cual vive.

En la Conferencia Mundial sobre Educación para todos de Jomtien (1990) ya se planteaba la necesidad de que cada persona pudiera satisfacer sus necesidades básicas de aprendizaje, debiendo recurrir para ello a unos contenidos básicos. Tras numerosos encuentros internacionales, y con la dificultad que plantea la diversidad de intereses (distintos puntos de partida, intereses políticos y económicos...), se han ido evaluando el grado de consecución de los objetivos propuestos en esta materia y su profundización.

El proyecto DeSeCo (1997), poniendo el acento en unas competencias clave, trabaja para identificarlas y determinar el nivel de competencias de jóvenes y adultos.

A nivel europeo otras organizaciones, como la mencionada EURYDICE, plantea la necesidad de un enfoque común para identificar las competencias clave, verdadero caballo de batalla 
para dar una respuesta educativa eficaz a los cambios que se están produciendo, de modo que se unifiquen esfuerzos y se puedan contrastar resultados y futuras proyecciones correctoras.

La respuesta por parte de los gobiernos no se hace esperar. El establecimiento de nuevos diseños curriculares incorpora unas competencias básicas como elemento imprescindible, para facilitar el que sus ciudadanos/as puedan ejercer sus derechos y deberes con plenitud, acordes a la sociedad cambiante a la que han de ir constantemente adaptándose.

De este modo, se sigue la línea establecida por la Comisión Europea de Educación, que considera unas competencias clave o destrezas básicas necesarias para el aprendizaje del individuo a lo largo de su vida, donde las competencias TIC que configuran la denominada alfabetización digital, como señala Marqués Graells (2000b), desempeñarán un papel destacado en el desarrollo personal, la inclusión social y la ciudadanía activa y el empleo.

Además, debemos preguntarnos por las expectativas de los receptores de estas políticas educativas (profesorado, equipos directivos, alumnado, ciudadanía en general) de modo que se sientan impulsores y partícipes activos. Puesto que son ellos los que en definitiva, determinarán el éxito ante los nuevos retos del binomio educación-TIC.

\section{6.- CONCLUSIONES.}

Se constata que las competencias básicas, son el fruto de un dilatado análisis internacional que se ha materializado como una línea de trabajo propuesta para seguir a través de los sistemas educativos nacionales, siendo entendidas como la capacidad para responder a las demandas y tareas de forma adecuada. Cada competencia se construirá a través de la combinación de habilidades cognitivas y prácticas, conocimiento, motivación, valores, actitudes, emociones y otros componentes sociales y conductuales.

Se plantea la adaptación a la globalización y al desplazamiento hacia las economías basadas en el conocimiento como un reto europeo, a partir de la Cumbre Extraordinaria de Europa en Lisboa (2000), proponiéndose la identificación de las competencias básicas para garantizar el acceso al aprendizaje en la sociedad de la innovación y el conocimiento, haciendo hincapié en que todo ciudadano deberá poseer la formación necesaria para vivir y trabajar en la sociedad de la información y el conocimiento.

Es en este plano donde las competencias digitales toman envergadura, materializándose en España en las últimas normativas educativas (LOE, 2006) con el establecimiento de la competencia $n^{\circ} 4$ : Tratamiento de la información y competencia digital que pasaría a englobar junto a las competencias lingüística, matemática y la cultural y artística el ámbito de la comunicación y la expresión.

Además estamos en condiciones de afirmar que las TIC juegan un papel fundamental en las competencias digitales, estando además sujetas a una continua y constante evolución, siendo necesario que sean integradas adecuadamente en los procesos educativos para lo que habrá que realizar los esfuerzos necesarios constantemente. Por tanto, el optimismo mostrado no debe eclipsar las dificultades existentes. Uno es consciente que la pretensión no es solucionar la problemática del aprendizaje de las competencias básicas en la enseñanza obligatoria, sino más bien orientar sobre las posibles acciones a seguir para actuar mejorando el proceso de enseñanzaaprendizaje.

Por otro parte, se está produciendo una constante reflexión sobre la necesidad de un cambio metodológico que dé respuesta a las necesidades TIC de la sociedad actual.

Finalmente, señalar que un buen aprendizaje de las competencias digitales ha de estar basado, en cualquier caso desde la reflexión, en un trabajo colaborativo e interdisciplinar de los diferentes ámbitos: educativo, social, pero también personal.

\section{7.- REFERENCIAS BIBLIOGRÁFICAS.}

Abad Amorós, R. (2005). Ciberseguridad: un compromiso de los Estados a partir de la Cumbre Mundial sobre la Sociedad de la Información (CMSI), Ginebra, 2003. En P. López López \& J. Gimeno Perelló (coords.), Información, conocimiento y bibliotecas en el marco de la globalización neoliberal (pp. 249-273). Gijón: Ediciones Trea..

Aviram, R. (2002). ¿Podrá la educación domesticar las TIC? Centro para el Futurismo en la Educación, (1-22), [en línea]. Ben Gurión: Universidad Ben Gurión. Disponible

en: http://web.udg.edu/tiec/ponencies/pon1.pdf. [Consulta: 2008, 17 octubre]. 
Bell, D. V. (1991). El advenimiento de la sociedad postindustrial: un intento de prognosis social. Madrid: Alianza.

Benito Morales, F. (2000). La alfabetización en información en centros de primaria y secundaria. En J. A. Gómez Hernández (coord.) Estrategias y modelos para enseñar a usar la información: guía para docentes, bibliotecarios y archiveros, (pp. 79-130), [en línea]. Murcia: KR. Disponible en: http://eprints.rclis.org/archive/00004672/03/ EMPEUIcap2.pdf., [Consulta: 2008, 23 de noviembre].

Castellano González, F. (2006). Las competencias básicas en educación. Revista de la Inspección, 2(1) [en línea]. Canarias: Consejería de Educación, Universidades, Cultura y Deportes. Disponible en: http://www.gobiernodecanarias.org/educacio n/udg/insp/Revista2006/content/articulo13/ar ticulo013.htm [Consulta: 2008, 2 de diciembre].

Castells, M. (1998). La era de la información (vol. II): El poder de la identidad. Madrid: Alianza Editorial.

Cebrián, J. L. (1999). Algunas certezas sobre la sociedad digital. En M. Caridad Sebastián (coord.). La sociedad de la información: Política, Tecnología e Industria de los contenidos. Madrid: Centro de Estudios Ramón Areces.

Chomsky, N. (1965). Aspects of the theory of syntax [en línea]. Cambridge: http://mitpress.mit.edu. Disponible en: http://books.google.es/books?hl=es\&lr=\&id $=$ u0ksbFqagU8C\&oi=fnd\&pg $=$ PR5\&dq $=\mathrm{Ch}$ omsky\&ots=5LsFqw0mZD\&sig=rLypc0Sx5zp6rRU9L-Rkm0Cbmk\#PPA44,M1, [Consulta: 2008, 10 de noviembre].

Comisión de las Comunidades Europeas (2005). Propuesta de Recomendación del Parlamento Europeo y del Consejo sobre las competencias clave para el aprendizaje permanente. Bruselas: Parlamento Europeo.

Díaz del Valle, S. \& Sánchez Baena, C. (2004). Claves para el éxito del Elearning. En, El profesorado ante el reto de las nuevas tecnologías en la Sociedad del Conocimiento. Congreso Internacional de la UGR y CSI-CSIF (1-12). Granada. Disponible http://www.uned.es/catedraunesco-
ead/publicued/Ponencia\%20Eriberto.pdf, [Consulta: 2008, 21 de noviembre].

Eurydice (2002). Las competencias clave. Un concepto en expansión dentro de la educación general obligatoria, [en línea]. Madrid: Ministerio de Educación, Cultura y Deporte. Disponible en: http://www.scribd.com/doc/486889/compete ncias-clave-Eurydice [Consulta: 2008, 2 de diciembre].

Gimeno Perelló, J. (2005). Revolución infotecnológica: el conocimiento transformado. En P. López López, P. \& J. Gimeno Perelló (coords.), Información, conocimiento y bibliotecas en el marco de la globalización neoliberal (pp. 215-248). Gijón: Ediciones Trea.

Laisner, F. (2000). Rèussir la formation par competentes. Montreal: Guérin.

Ley Orgánica 2/2006, de 3 de mayo, de Educación (BOE n $\left.{ }^{\circ} 106\right)$.

Ley Orgánica 5/2002, de 19 de junio, de las Cualificaciones y de la Formación Profesional (BOE ${ }^{\circ}$ 147).

Linares, J. (1995). Autopistas inteligentes. Madrid: Fundesco.

López, J. (2006). Las competencias básicas del currículo en la LOE [en línea]. Granada: V Congreso Internacional "Educación y Sociedad”. Disponible en: http://congreso.codoli.org/conferencias/JuanLopez.pdf. [Consulta: 2008, 18 de octubre]

Majó, J (2003). Nuevas tecnología y educación. Conferencia presentada en la Universitat Oberta de Catalunya [en línea]. Barcelona: UAB. Disponible en: http://www.uoc.es/web/esp/articles/joan maj o.html. [Consulta: 2008, 3 de diciembre].

Marquès Graells, P. (2000a). Impacto de las TIC en educación: funciones y limitaciones. DIM, [en línea]. Disponible en: http://dewey.uab.es/PMARQUES/siyedu.ht m. [Consulta: 2008, 15 de noviembre].

Marquès Graells, P. (2000b). Nueva cultura, nuevas competencias para los ciudadanos. La alfabetización digital. Roles de los estudiantes hoy. DIM [en línea]. Disponible en:

http://dewey.uab.es/PMARQUES/competen. htm [Consulta: 2008, 20 de noviembre].

Marqués Graells, P. (2008). La escuela del 2015. Las competencias TIC del docente. 
Contextualizadas desde el documento: "las competencias digitales de los docentes". TICEMUR 2008. Disponible en: http://dewey.uab.es/pmarques/docs/ticemurf oro3.doc, [Consulta: 2008. 13 de octubre].

Noriega Álvarez, J. \& Muñoz González, A. (1996). Técnicas básicas para elaborar el programa de dirección. Madrid: Escuela Española.

OECD (2005). La definición y selección de competencias clave [en línea]. Paris. Disponible en: http://www.deseco.admin.ch/bfs/deseco/en/i ndex/03/02.html [Consulta: 2008, 15 de noviembre].

Organización de Estados Iberoamericanos para la Educación, la Ciencia y la Cultura. Declaración Mundial sobre educación para todos "Satisfacción de las necesidades básicas de aprendizaje” [en línea]. Disponible en: http://www.oei.es/efa2000jomtien.htm, [Consulta: 2008, 18 de noviembre].
Organización de las Naciones Unidas (2005). Cumbre Mundial sobre la Sociedad de la Información Ginebra 2003-Túnez 2005. Declaración de Principios [en línea]. Disponible en: http://www.itu.int/dms pub/itus/md/03/wsis/doc/S03-WSIS-DOC0004!!MSW-S.doc . [Consulta: 25 de noviembre de 2008].

Sarramona, J. (2008). Las competencias básicas y su incidencia sobre el curriculum escolar. En P. García Contreras (coord.), Competencias básicas y evaluación. C.P.R. Cartagena: (inédito).

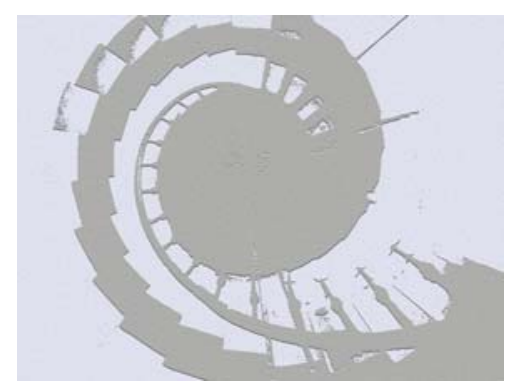

